\title{
Electromagnetic Resonant Properties of Metal-Dielectric-Metal (MDM) Cylindrical Microcavities
}

\author{
Hang $\mathrm{HENG}^{1 *}$ and Rong $\mathrm{WANG}^{2,3}$ \\ ${ }^{1}$ Center for Analysis and Testing, Nanjing Normal University, Nanjing, 210097, China \\ ${ }^{2}$ Department of Neurosurgery, Nanjing Drum Tower Hospital, Nanjing, 210008, China \\ ${ }^{3}$ The Affiliated Hospital of Nanjing University Medical School, Nanjing, 210008, China \\ *Corresponding author: Hang HENGＥ-mail: 3079576@qq.com
}

\begin{abstract}
Optical metamaterials can concentrate light into extremely tiny volumes to enhance their interaction with quantum objects. In this paper, a cylindrical microcavity based on the Au-dielectric-Au sandwiched structure is proposed. Numerical study shows that the cylindrical microcavity has the strong ability of localizing light and confining $10^{3}--10^{4}$-fold enhancement of the electromagnetic energy density, which contains the most energy of the incoming light. The enhancement factor of energy density $G$ inside the cavity shows the regularities as the change in the thickness of the dielectric slab, dielectric constant, and the radius of gold disk. At the normal incidence of electromagnetic radiation, the obtained reflection spectra operate in the range from $4.8 \mu \mathrm{m}$ to $6 \mu \mathrm{m}$ and with the absorption efficiency $C\left(C=1-R_{\min }\right)$, which can reach $99 \%$ by optimizing the structure's geometry parameters, and the dielectric constant. Due to the symmetry of the cylindrical microcavities, this structure is insensitive to the polarization of the incident wave. The proposed optical metamaterials will have potential applications in the surface enhanced spectroscopy, new plasmonic detectors, bio-sensing, solar cells, etc.
\end{abstract}

Keywords: Microcavity; metal-semiconductor-metal; metamaterial

Citation: Hang HENG and Rong WANG, "Electromagnetic Resonant Properties of Metal-Dielectric-Metal (MDM) Cylindrical Microcavities," Photonic Sensors, 2017, 7(2): 148-156.

\section{Introduction}

Recently, surface plasmon polaritons (SPPs) and localized surface plasmon polaritons (LSPs) are widely researched in the basis theory of optical metamaterials $[1,2]$. Metal-dielectric photonic band gap structures [3, 4] are usually used to excite SPPs and LSPs. They have many extraordinary optical properties, such as surface enhanced spectroscopy [5, 6], enhanced transmission [7-9], sensitive bio-sensing $[10,11]$, negative refractive index [12-14], double negative materials [15], and high resolution imaging $[16,17]$. The metallic gratings consisting of narrow slits [18] may become transparent for extremely broad bandwidths under the oblique incidence. In addition, ultrathin, broadband, highly efficient [19, 20], and freely tunable [21] metamaterial-based polarization converters have been realized. In the visible and infrared range, as the wavelength increases, the penetration depth into the dielectric layer increases. At the same time, the ohmic loss of metal layers reduces reversely [22]. In particular, the ohmic loss is very low in the far infrared region, where it has a

Received: 12 September 2016 / Revised: 11 November 2016

(C) The Author(s) 2017. This article is published with open access at Springerlink.com

DOI: $10.1007 / \mathrm{s} 13320-017-0379-3$

Article type: Regular 
perfect reflection characteristic. Because of these features, we can break the diffraction limitation and confine light within a sub-wavelength volume [23]. So far, confining light within a sub-wavelength volume has become one of the hot issues in the optical metamaterial research. In the terahertz region, dimensions can be reduced to extreme subwavelength [23, 24]. Furthermore, Fevillet-Palma et al. [25] studied the near-field enhancement properties of the antenna in the terahertz region and originally calculated the electromagnetic energy density in the cavities. However, the cuboid microcavities have the lower absorption efficiency, and this cuboids cavity is sensitive to the polarization of the incident wave.

In this work, we first proposed metal-dielectric-metal (MDM) cylindrical microcavities and numerically calculated the electromagnetic energy density in the cavities and the concentration mechanism and the absorption properties of the cylindrical microcavities through the mathematical model by high frequency structure simulator (HFSS, commercial software). Compared with the literature [25], we got a higher absorption efficiency $C$. Because the gold disk is of circular symmetry, the structure was insensitive to the polarization of the incident light. The proposed model and calculation method will play a guidance role in the optimization of substrates for the surface enhanced spectroscopy.

\section{Simulation and structures}

The designed MDM structure is simulated by HFSS, a finite element method based program. The MDM cylindrical cavities are periodically arranged in a square lattice in the $X-Y$ plane, as shown in Fig. 1(a). Figure 1(b) is the schematic of a unit cell of the cylindrical cavity array. A dielectric layer is sandwiched between two metallic layers: the top layer is a gold disk, and the bottom layer is a gold ground layer. The deep black area between the gold disk and gold ground layer in Fig. 1(b) is the cylindrical microcavity formed between a gold disk

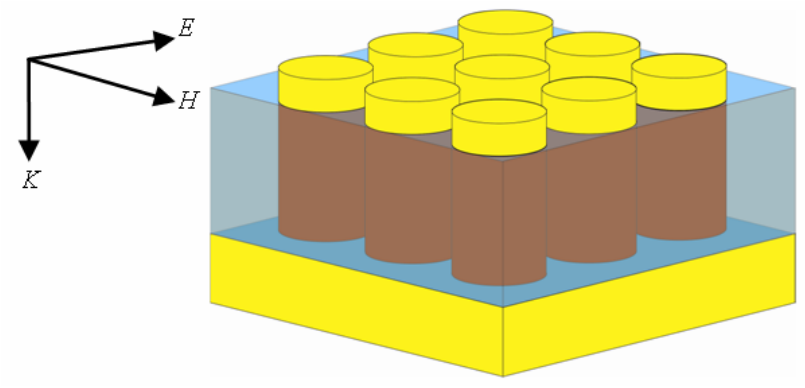

(a)

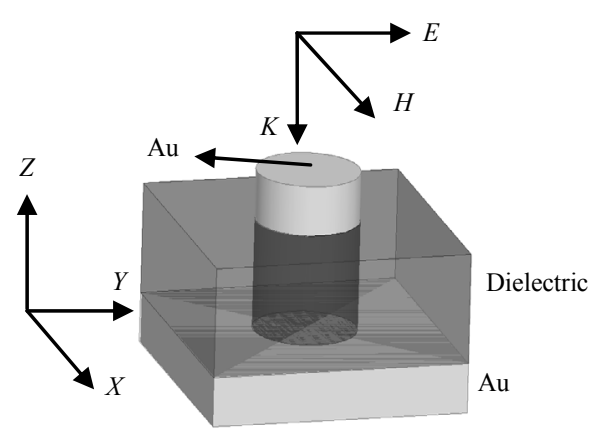

(b)

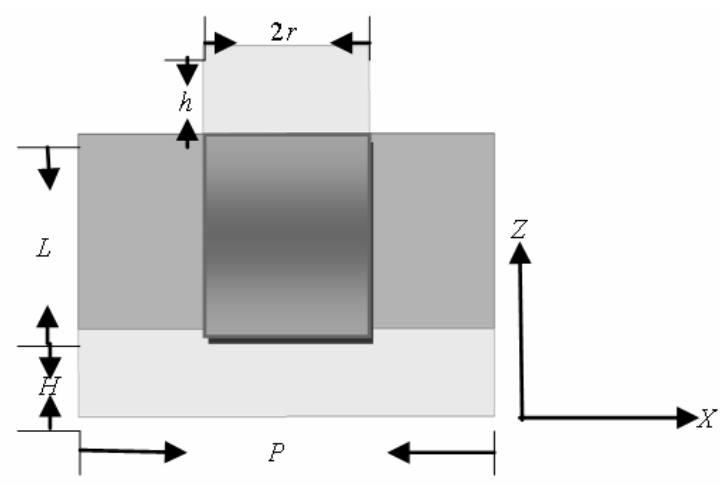

(c)

Fig. 1 Schematic of the designed MDM structure: (a) schematic of the cylindrical cavity array, (b) schematic geometry of a unit cell of the cylindrical microcavity at the normal incident TM plane wave (the top is a gold disk, the middle is a dielectric layer, the bottom is a gold plane, and the deep black area between the gold disk and gold ground layer is the cylindrical microcavity), and (c) cross section view of the cylindrical microcavity in the $Y-Z$ plane $(r, h, L$, and $P$ represent the gold disk radius, thickness of the gold disk, height of the microcavity, and period, respectively).

and a gold ground layer. If the thickness $L$ of the dielectric layer is smaller than the wavelength, such a structure supports a $\mathrm{TM}_{100}$ mode [25]. Figure 1(c) shows the cross section of the cavity in the $Y$ - $Z$ plane, and the structural parameters are shown in the figure. The thickness of the dielectric layer is chosen as $L$, and $h$ and $r$ are defined as the thickness and radius 
of the gold disk, respectively. In this paper, the thickness of the gold ground layer is chosen as $0.45 \mu \mathrm{m}$. The gold ground layer has two significant functions. One is to serve as an optical mirror. As the thickness is greater than the penetration depth of the incident light, the transmittance is close to zero. The other is to couple with the gold disk and gold ground layer to create electric and magnetic dipoles which extremely concentrate electromagnetic energy into the microcavities. Period $P$ is $3 \mu \mathrm{m}$. The orientations of the electric field $(E)$, the magnetic field $(H)$, and the wave vector $(K)$ are shown in Fig. 1(b). The incident angle is 0 . The boundary conditions are set as the perfect electric conductor and perfect magnetic conductor on two-pair faces. The dielectric permittivity of gold is modeled by using the Drude model [26]

$$
\varepsilon=1-\frac{\omega_{P}^{2}}{\omega^{2}+\mathrm{i} \omega \gamma}
$$

with the plasma frequency $\omega_{P}=1.37 \times 10^{16} \mathrm{rad} / \mathrm{s}$ and scattering frequency $\gamma=4.08 \times 10^{13} \mathrm{rad} / \mathrm{s}$.

\section{Results and discussion}

In order to study the resonant characteristics of the cylindrical microcavity, we calculate the reflectivity spectra $R(\omega)=\left|S_{11}(\omega)\right|^{2}$ at the normal incident TM plane wave. As shown in Fig. 2, the resonant cavity mode appears as a reflection dip in the spectra. Moreover, the grating period is kept subwavelength, so that the only contribution to the reflected signal is the specular reflection (0th diffraction order) [25]. We also calculate the transmission of the structure shown in Fig. 1 and obtain near zero transmission for all geometry parameters (the transmission spectra are not given here). According to the equation

$$
T+R+C=1
$$

where $T, R$, and $C$ are the transmission, reflection, and absorption, respectively. Due to $T=0$, the minimum of reflection appears as the maximum of absorption. If we define $C=1-R_{\min }$, where $R_{\min }$ is the minimum reflectivity at the resonant frequency, a larger $C$ indicates a stronger absorption efficiency.

\subsection{Absorption of the configuration}

Figure 2(a) is the reflectivity spectra of the cylindrical microcavity in the MDM structure at the normal incident with variable dielectric constant $\varepsilon$, here $L=1 \mu \mathrm{m}, h=0.45 \mu \mathrm{m}, H=0.45 \mu \mathrm{m}$, and $P=3 \mu \mathrm{m}$. When $\varepsilon$ changes from 10 to 14 (with a step of 1), the resonant frequency is around $60.3 \mathrm{THz}, 57.5 \mathrm{THz}$, $54.95 \mathrm{THz}, 52.9 \mathrm{THz}$, and $51 \mathrm{THz}$, and $C$ is $85 \%$, $88 \%, 97 \%, 99 \%$, and $97 \%$, respectively. It can be seen that the reflection peaks show red-shift with an increase in the dielectric constant, and this means that the greater the dielectric constant is, the greater the near-field coupling is, which reaches an agreement with that in [27]. This is because the change in the effective dielectric permittivity $[\varepsilon,(\omega)]$ of the system results in a shift of the electric resonance frequency. And the rate of red-shift is proportional to the dielectric constant. Figure 2(b) shows the reflectivity spectra as a function of $L$. As shown in Fig. 2(b), the resonant frequency mainly locates in the range of $(53.7 \pm 0.75) \mathrm{THz}$, whose dependence on $L$ is relatively weak [27]. In contrast, the dielectric constant $\varepsilon$ has much more greater impacts on the electric dipole resonances due to the excitement of the external electric field than $L$. At $L=0.98 \mu \mathrm{m}, C$ is $96 \%$. When $L$ increases to $0.99 \mu \mathrm{m}$, $C$ reaches the maximum $99 \%$. A further increase in $L$ will reduce $C$. At $L=1.00 \mu \mathrm{m}, C$ declines to $98 \%$. When $L$ changes from $1.01 \mu \mathrm{m}$ to $1.03 \mu \mathrm{m}$, the absorption efficiency inside the cavity continues to fall. The results show that the absorption efficiency inside the cavity is closely related to $L$. Figure 2(c) presents the reflectivity spectra as a function of $r$, here $L=1 \mu \mathrm{m}, h=0.45 \mu \mathrm{m}, H=0.45 \mu \mathrm{m}$, and $P=3 \mu \mathrm{m}$. When $r$ increases from $0.4 \mu \mathrm{m}$ to $0.9 \mu \mathrm{m}$ (with a step of $0.1 \mu \mathrm{m})$, the resonant frequency locates at $56.255 \mathrm{THz}, 54.255 \mathrm{THz}, 53.75 \mathrm{THz}, 53.355 \mathrm{THz}$, $53.25 \mathrm{THz}$, and $53.15 \mathrm{THz}$, respectively and $C$ equals to $71 \%, 89 \%, 99 \%, 88 \%, 69 \%$, and $50 \%$, respectively. As shown in Fig. 2(c), the resonant 
peaks show red-shift with an increase in the radius, which has a similar trend as Fig. 2(a), and this near-field coupling phenomenon was reported in
[25]. The results also show that the absorption efficiency inside the cavity is sensitive to the radius of the gold disk.

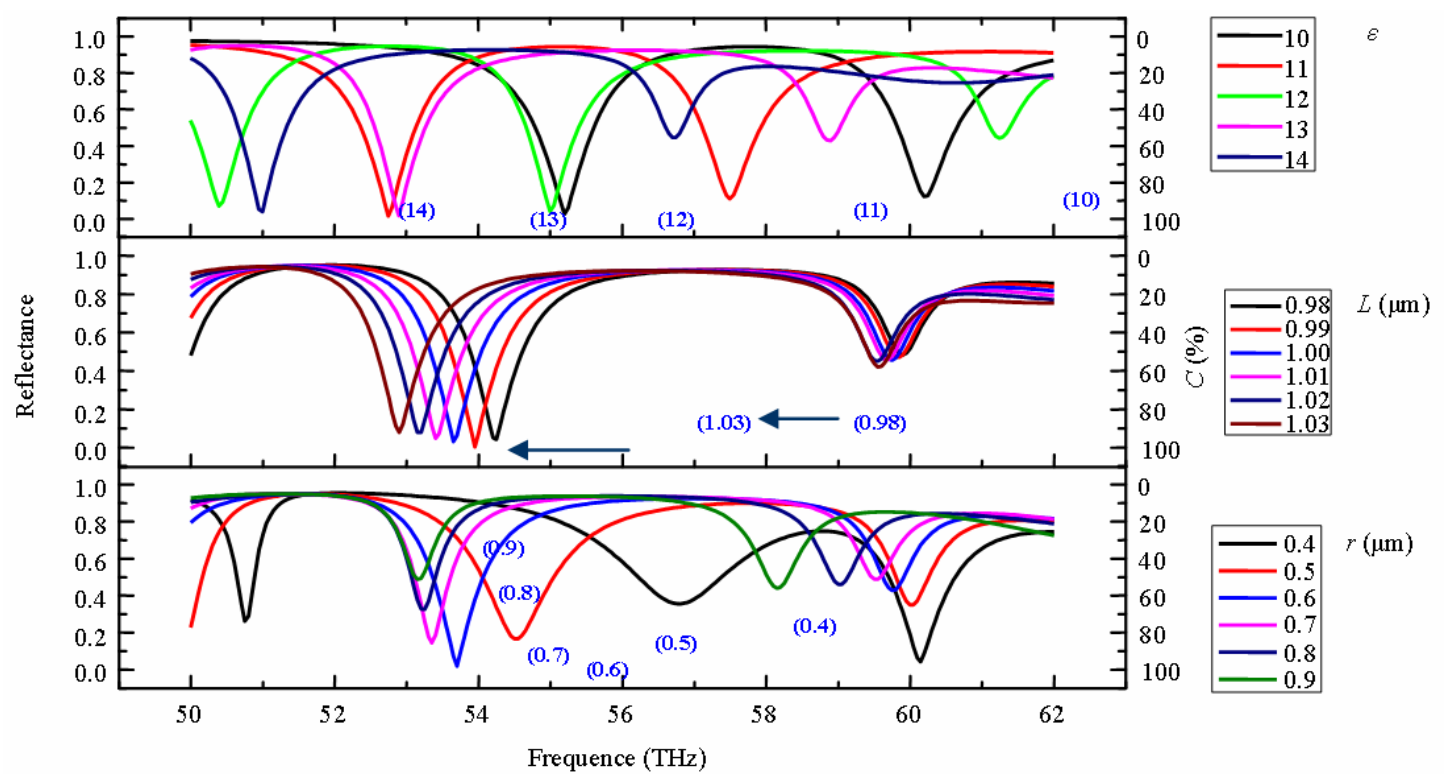

Fig. 2 Reflection spectra of the cylindrical microcavity in the MDM structure at the normal incident TM plane wave: (a) reflectivity spectra with the change in the dielectric constant $\varepsilon, L=1 \mu \mathrm{m}, h=0.45 \mu \mathrm{m}, H=0.45 \mu \mathrm{m}$, and $P=3 \mu \mathrm{m}$, (b) reflection spectra with the change in $L, r=0.6 \mu \mathrm{m}, H=0.45 \mu \mathrm{m}$, and $P=3 \mu \mathrm{m}$, and (c) reflectivity spectra with the change in $r, L=1 \mu \mathrm{m}, h=0.45 \mu \mathrm{m}, H=0.45 \mu \mathrm{m}$, and $P=3 \mu \mathrm{m}$.

\subsection{Localizing features}

As reported in [25], the energy of the incidence wave is dissipated by both the metallic and the dielectric losses, and the large part of the energy can been localized in the dielectric microcavity. In order to study the regularity of the energy density in the condition of different parameters, we calculate the energy density of the electromagnetic field inside the cylindrical cavity with the change in the dielectric constant, the thickness of the dielectric slab, and the radius of the gold disk. Because of the energy conservation, the peak electrical energy is equal to the average total energy inside the cavity, and the solution is achieved by integrating $E \cdot E^{*}$ within the volume

$$
E_{d}=\int_{V} \frac{E \cdot E^{*}}{V} d v
$$

where $V$ is the volume of the cavity, $E_{d}$ is the energy density inside the cavity, and $E$ represents the electric field vector inside the cavity. Firstly, we calculate the energy density $E_{d 1}$ inside the dielectric cavity in the MDM structure. Then, we calculate the energy density $E_{d 2}$ inside the vacuum cavity without the MDM structure, whose shape and size are the same as the dielectric cavity. We define $G=E_{d 1} / E_{d 2}$ as the enhancement factor of the energy density. A cavity with too big or small volume will affect the electromagnetic (EM) energy absorption and confinement inside the cavity [23]. Only when the parameters of the material and geometry are optimized, the impedance of the structure matching to the impedance of vacuum will the extreme confinement of the EM field and strong absorption inside the cavities appear. Figure 3(a) shows the enhancement factor of the energy density $G$ as a function of $\varepsilon$. It can be seen that the enhancement factor $G$ increases with an increase in the dielectric constant $\varepsilon$. Because the near-field coupling between the top and bottom metal layers become stronger [27], the ability of localizing the light inside the dielectric cavities is strengthened as shown in Fig. 3(a). Figure 3(b) shows the energy density ratio $G$ as a function of the thickness of the dielectric slab $L$. 
As shown in Fig. 3(b), when $L$ changes from 0.98 $\mu \mathrm{m}$ to $0.99 \mu \mathrm{m}, G$ shows the trend of growth. When $L$ increases to $0.99 \mu \mathrm{m}, G$ reaches the maximum 4180. A further increase in $L$ will reduce $G$. At $L=1.03 \mu \mathrm{m}, G$ declines to 2945. Figure3(b) reveals very important field spreading in the plane of the metallic strip [23] when $L$ changes from $0.99 \mu \mathrm{m}$ to $0.98 \mu \mathrm{m}$. So the enhancement factor $G$ is sensitive to $L$, and the most optimal $G$ should also be related to the effective dielectric permittivity $[\varepsilon(\omega)]$ of the system. Figures 4(a) and 4(b) present the energy density ratio $G$ and absorption efficiency $C$ as a function of the radius $r$ of the gold disk, respectively. From Fig.4(a), we can see that $G$ shows the trend of increase first and when $r=0.5 \mu \mathrm{m}, G$ reaches the maximum 3314. Afterwards, $G$ begins to decrease. As shown in Fig. 4(b), their variation trend is the same as that shown in Fig.4(a). Their difference is that $C$ reaches the maximum $97 \%$ when $r=0.6 \mu \mathrm{m}$. It can be seen that the maximum of $C$ does not indicate the maximum of the energy density ratio. To explain this phenomenon, we use the following model (4):

$$
\frac{\varepsilon\left|E_{Z 0}\right|^{2}}{\left|E_{\text {in }}\right|^{2}}=\frac{\lambda_{\text {res }} \Sigma}{2 \pi V} Q_{\text {ohm }} C \cos \theta
$$

where $\varepsilon$ is the dielectric constant of the middle layer, $V=\pi r^{2} L$ is the volume of the cylindrical resonator, $E_{\text {in }}$ is the electric field amplitude of the incoming wave, $E_{Z 0}$ is the amplitude of the mode resonantly excited in the microcavity, and $\theta$ is the incident angle. For the data reported here, we have $\cos \theta=1$, $\Sigma=P^{2}$, and $Q_{\text {ohm }}$ is the quality factor $Q$ of the Ohmic loss. When $r=0.6 \mu \mathrm{m}, C=0.97, G=3232, \quad r=0.5$ $\mu \mathrm{m}$, and $R=3314, C$ is 0.85 . Because $Q_{\text {ohm }}$ approximately equals in the bands studied here, we can get $\frac{\varepsilon\left|E_{Z 0}\right|^{2}}{\left|E_{\text {in }}\right|^{2}}=\lambda_{\text {res }} C / V$, indicating that the energy density ratio inside the cavity is decided by $\lambda_{\text {res }} C / V$. At $r=0.6 \mu \mathrm{m}$, its $\lambda_{\text {res }} C$ is greater than that at $r=0.5 \mu \mathrm{m}$. As the volume plays the most important role in $R, R$ reaches the maximum (3314) at $r=0.5 \mu \mathrm{m}$.

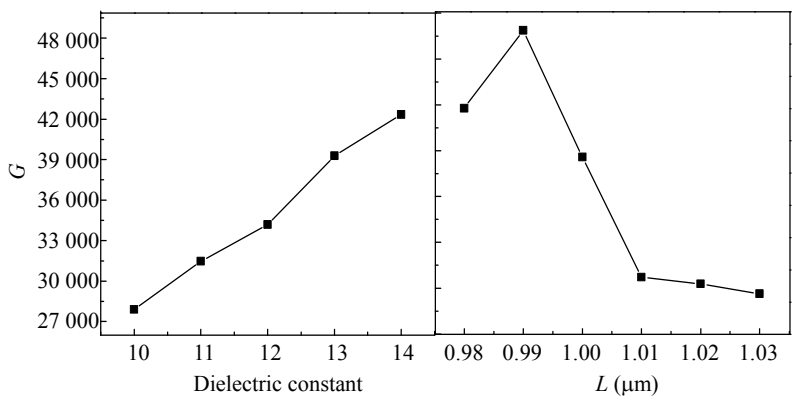

(a)

(b)

Fig. 3 Energy density ratio $G$ as the dielectric constant $\varepsilon$ and the thickness $L$ of the dielectric slab: (a) energy density ratio $G$ as a function of the dielectric constant $\varepsilon$ and (b) energy density ratio $G$ as a function of the thickness $L$ of the dielectric slab.

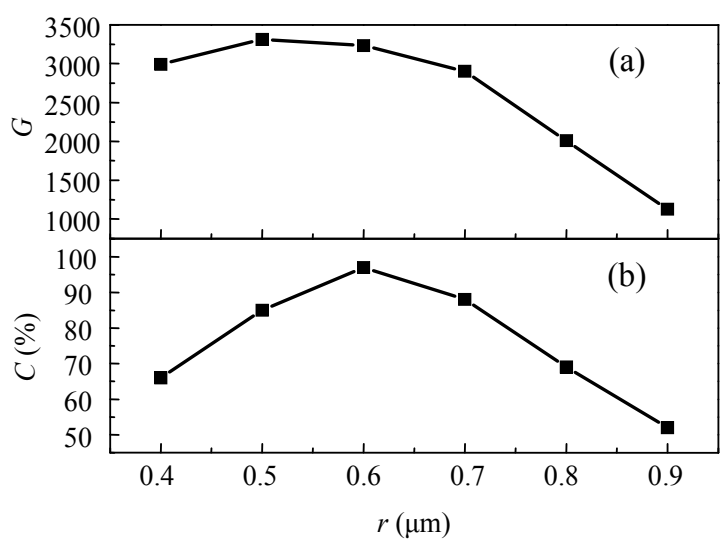

Fig. 4 Energy density ratio $G$ and absorption efficiency $C$ as a function of the radius $r$ of the gold disk: (a) energy density ratio $G$ as a function of the radius $r$ of the gold disk and (b) absorption efficiency $C$ as a function of the radius $r$ of the gold disk.

\subsection{Physical mechanism}

To explore the physical mechanism of the extreme confinement and perfect absorption inside the cavity, the distributions of the EM field intensity and current at the resonant mode $(f=53.7 \mathrm{THz})$ are simulated, as shown in Fig. 5. Figure 5(a) shows the amplitude of the electric field in the middle of the dielectric layer in the $X-Y$ plane. The gray circle represents the cylindrical microcavity. A standing wave pattern is clearly shown in Fig. 5(a). The amplitude of the electric field can be approximated by the expression

$$
|E| \sim \cos \frac{\pi X}{\Delta x}(0 \leq X<r) .
$$

At $r=0,|E|$ reaches its maximum, that is, the energy is strongly localized around the center of the cylindrical microcavity. Figure 5(b) presents the 
amplitude of the electric field in the middle of the dielectric layer in the $Y-Z$ plane. In this figure, the white area represents the metal, the middle layer is dielectric, and the EM field is highly localized between the two metal layers, which can be approximated as a standing wave too. The confining mechanism, which arises from the impedance mismatch between the metal-metal and single metal regions [24], is responsible for the formation of the standing wave patterns. The perfect absorbing

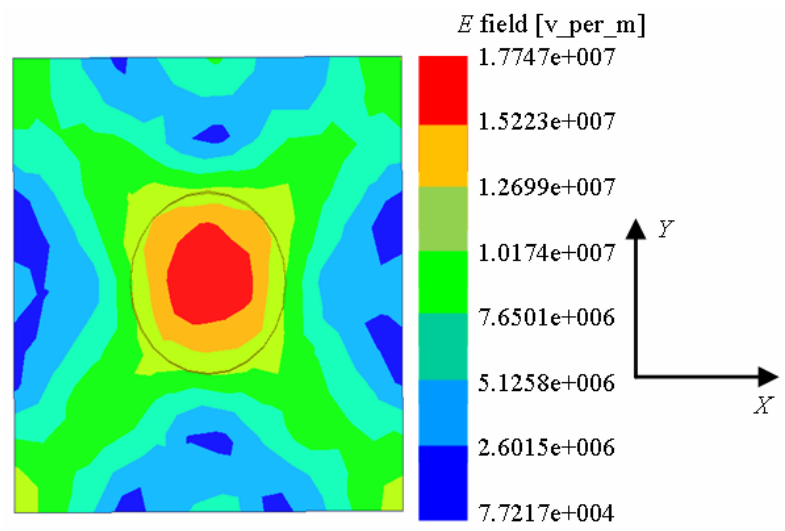

(a)

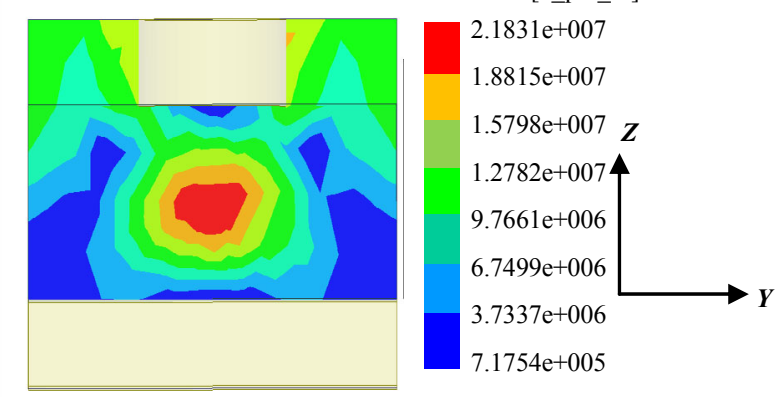

(b)

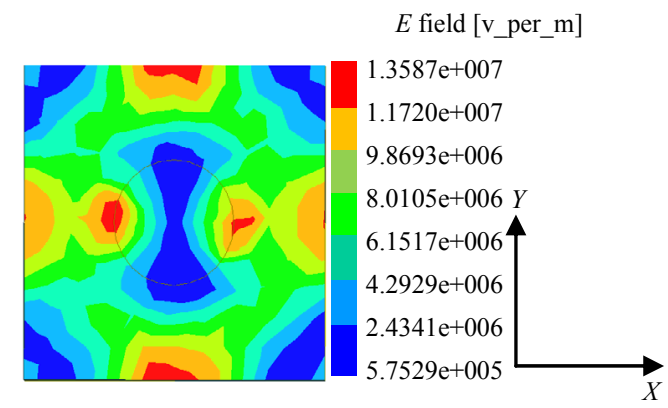

(c)

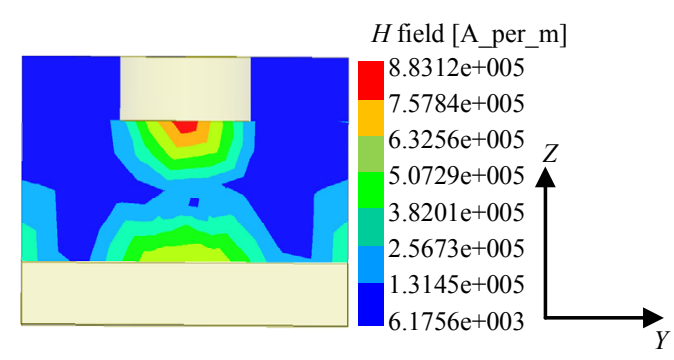

(d)

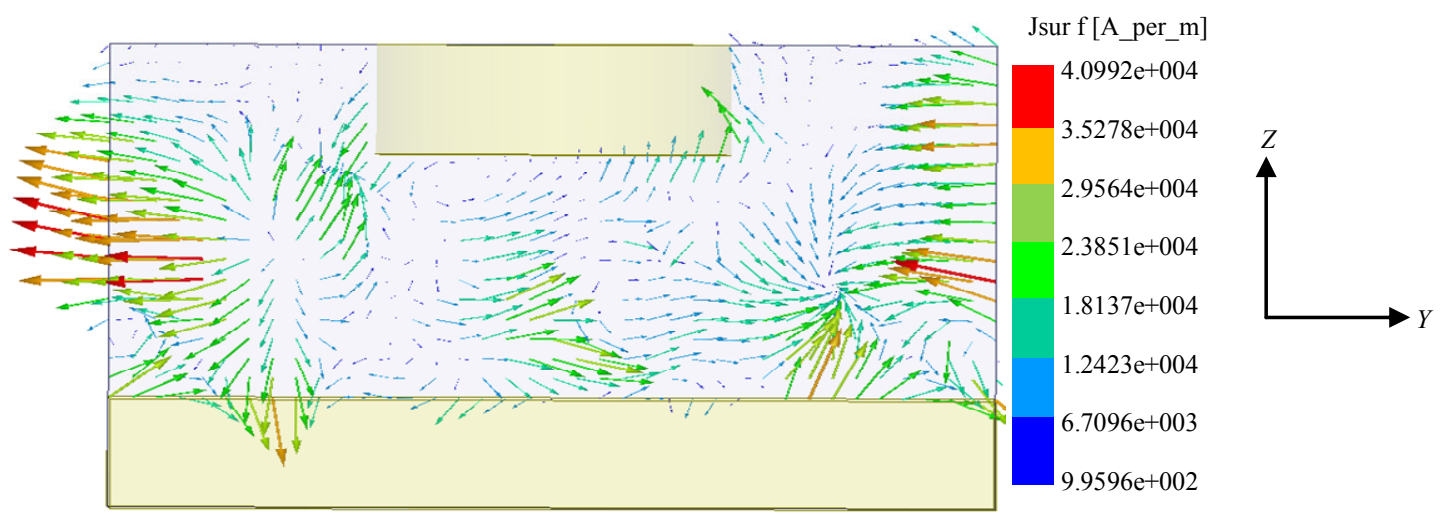

(e)

Fig. 5 Distributions of the EM field intensity and current within cylindrical cavities at the normal incident TM plane wave: (a) amplitude of the electric field in the middle of the dielectric layer in the $X$-Y plane at $f=53.7 \mathrm{THz}$, (b) amplitude of the electric field in the middle of the dielectric layer in the $Y-Z$ plane at $f=53.7 \mathrm{THz}$, (c) amplitude of the electric field on the top surface of the dielectric layer at $f=53.7 \mathrm{THz}$, (d) amplitude of magnetic field in the middle of the dielectric layer in the $Y-Z$ plane at $f=53.7 \mathrm{THz}$, and (e) distribution of the current in the middle of the dielectric layer in the $Y-Z$ plane at $f=53.7 \mathrm{THz}$. 
characteristics may be explained by Figs.5(c)-5(e). Figure 5(c) shows the amplitude of the electric field on the top surface of the dielectric layer, and here the gray circle represents the position of the gold disk. As indicated in Fig.5(c), the distribution of the electric field mainly concentrates around the gold disk, forming a square area. However, the amplitude of the electric field in the center of the gold disk is weaker than that in the surrounding. Meanwhile, it can be seen clearly that six "hot spots" symmetrically locate in the four edges of the square area and both sides of the gold disk in the $Y$-axis direction. In this case, the gold disk can be regarded as the electric dipole resonator by the external electric field excitation. A change in the disk diameter will result in a shift of the electric resonance frequency and hence result in a change in the effective dielectric permittivity $[\delta(\omega)]$ of the system. Figure 5(d) shows the distribution of the current in the middle of the dielectric layer in the $Y-Z$ plane. The white areas in this figure are the two metal layers. The magnetic field distribution shown in Fig. 5(d) clearly indicates the localization of the magnetic field between two metal layers, and the formation of the magnetic dipole resonance. A change in the dielectric layer thickness mainly affects the magnetic resonance and the effective magnetic permeability $[\mu(\omega)]$ of the system. By optimizing the parameters of the top gold layer and dielectric layer thickness, we can tune $\omega$ of the $\varepsilon$ and $\mu$ resonances. When we achieve $\varepsilon=\mu$ $\left(Z=\sqrt{\frac{\varepsilon(\omega)}{\mu(\omega)}}=1\right)$, an impedance near the free space value is formed. With strongly coupling the incident radiation to the resonant structure and perfect absorbance being theoretically possible, it can only occur when the metal-metal layer is impedance-matched to the free space [28]. The distribution of the current in the middle of the dielectric layer in the $Y-Z$ plane is shown in Fig. 5(e), here the arrows in the dielectric cavity represent the magnitude and direction of the current. Figure 5(e) clearly indicates that the currents on the disk and the ground plane are opposite, forming a circulating current known as a magnetic resonance, which results in an artificial magnetic moment that interacts strongly with the magnetic field of the incident light. If the electric and magnetic dipole resonances occur at the same frequency, then there will be a strong localization of the electromagnetic energy inside the cylindrical microcavity. To realize the high confinement and perfect absorption, the optical metamaterials are usually engineered by simultaneously minimizing the reflectance with perfect impedance matching and eliminating the transmittance by maximizing material losses.

\section{Conclusions}

We have numerically studied the electromagnetic energy density and absorption efficiency inside the MDM cylindrical microcavities in the mid-infrared range. As the change in the thickness of the dielectric slab, dielectric constant, and the radius of gold disk, $G$ and $C$ present some important regularities for us to modulate the resonance frequency and strengthen electromagnetic energy density. The enhancement factor $G$ increases with an increase in the dielectric constant $\varepsilon$. When $L$ changes from $0.98 \mu \mathrm{m}$ to $1.03 \mu \mathrm{m}, G$ increases first and then decrease. When $L=0.99 \mu \mathrm{m}, G$ reaches the maximum 4180. At $L=1 \mu \mathrm{m}, H=0.45 \mu \mathrm{m}, h=$ $0.45 \mu \mathrm{m}$, and $r=0.6 \mu \mathrm{m}, C$ is 0.97 , and $R$ is 3232 . When $r=0.5 \mu \mathrm{m}$ with other conditions unchanging, $C$ is 0.85 , and $R$ is 3314 . It can be seen that the maximum of $C$ does not indicate the maximum of the energy density ratio. This kind of strong absorption and high localization makes the proposed structures have broad applications in the areas such as the surface enhanced spectroscopy, new plasmonic detectors, bio-sensing, and solar cells.

\section{Acknowledgment}

The authors gratefully acknowledge the financial 
support provided to this study by the Program of Natural Science Research of Jiangsu Higher Education Institutions of China (Grant No. 14KJB140005).

Open Access This article is distributed under the terms of the Creative Commons Attribution 4.0 International License (http://creativecommons.org/ licenses/by/4.0/), which permits unrestricted use, distribution, and reproduction in any medium, provided you give appropriate credit to the original author(s) and the source, provide a link to the Creative Commons license, and indicate if changes were made.

\section{References}

[1] G. Q. Liu, F. L. Tang, L. Li, L. X. Gong, and Z. Q. Ye, "Concentration detection of quantum dots in the visible and near-infrared range based on surface plasmon resonance sensor," Materials Letters, 2011, 65(12): 1998-2000.

[2] Y. Y. Yang, Y. L. Zhang, F. Jin, X. Z. Dong, and X. M. Duan, "Steering the optical response with asymmetric bowtie 2-color controllers in the visible and near infrared range," Optics Communications, 2011, 284(13): 3474-3478.

[3] D. Owens, C. Fuentes-Hernandez, and B. Kippelen, "Optical properties of one-dimensional metaldielectric photonic band-gap structures with low index dielectrics," Thin Solid Films, 2009, 517(8): 2736-2741.

[4] J. Y. Seo, S. Cho, H. Lim, and S. Lee, "Optical and structural properties of metal-dielectric photonic band gap structures," Current Applied Physics, 2006, 6(6): 62-66.

[5] P. Z. El-Khoury, E. J. Bylaska, and W. P. Hess, "Time domain simulations of chemical bonding effects in surface-enhanced spectroscopy," Journal of Chemical Physics, 2013, 139(17): 174303-1-174303-5.

[6] C. L. Du, C. J. Du, Y. M. You, Y. Zhu, S. L. Jin, C. J. $\mathrm{He}$, et al., "Numerically investigating the enhanced Raman scattering performance of individual Ag nanowire tips," Applied Optics, 2011, 50(25): 4922-4926.

[7] L. Zhu, L. Dong, F. Y. Meng, J. H. Fu, and Q. Wu, "Influence of symmetry breaking in a planar metamaterial on transparency effect and sensing application," Applied Optics, 2012, 51(32): 7794-7799.

[8] T. W. Ebbesen, H. J. Lezec, H. F. Ghaemi, T. Thio, and P. A. Wolff, "Extraordinary optical transmission through sub-wavelength hole arrays," Nature, 2001, 86(6): 1114-1117.
[9] H. F. Ghaemi, T. Thio, and D. E. Grupp, "Surface plasmons enhance optical transmission through subwavelength holes," Physical Review B, 1998, 58(11): 357-368.

[10] A. J. Haes, S. L. Zou, G. C. Schatz, and R. P. Van Duyne, "Nanoscale optical biosensor: short range distance dependence of the localized surface plasmon resonance of noble metal nanoparticles," Journal of Physical Chemistry B, 2003, 108(22): 6961-6968.

[11] A. V. Kabashin, P. Sergiy, and A. N. Grigorenko, "Phase and amplitude sensitivities in surface plasmon resonance bio and chemical sensing," Optics Express, 2009, 17(23): 21191-21204.

[12] R. A. Shelby, D. R. Smith, and S. Schultz, "Experimental verification of a negative index of refraction," Science, 2001, 292(292): 77-79.

[13] J. Wu, B. Ng, S. P. Turaga, M. B. H. Breese, S. A. Maier, M. Hong, et al., "Free-standing terahertz chiral meta-foils exhibiting strong optical activity and negative refractive index," Applied Physics Letters, 2013, 103(14): 141106-1-141106-4.

[14] G. Dolling, M. Wegener, C. M. Soukoulis, and S. Linden, "Negative-index metamaterial at $780 \mathrm{~nm}$ wavelength," Optics Letters, 2007, 32(1): $53-55$.

[15] F. L. Zhao, B. A. Kamil, C. Evrim, and O. Ekmel, "Complementary chiral metamaterials with giant optical activity and negative refractive index," Applied Physics Letters, 2011, 98(16): 161907-1-161907-3.

[16] R. Tomer, L. Ye, B. Hsueh, and K. Deisseroth, "Advanced clarity for rapid and high-resolution imaging of intact tissues," Nature Protocols, 2014, 9(7): 1682-1697.

[17] T. Taubner, D. Korobkin, Y. Urzhumov, G. Shvets, and R. Hillenbrand, "Near-field microscopy through a SiC superlens," Science, 2006, 313(5793): 1595-1595.

[18] F. I. Baida, M. Boutria, R. Oussaid, and D. Van Labeke, "Enhanced-transmission metamaterials as anisotropic plates," Physical Review B, 2011, 82(3): 2109-2119.

[19] X. R. Huang, R. W. Peng, and R. H. Fan, "Making metals transparent for white light by spoof surface plasmons," Physical Review Letters, 2010, 105(24): 119-127.

[20] R. H. Fan, R.W. Peng, X. R. Huang, J. Li, Y. Liu, Q. $\mathrm{Hu}$, et al., "Transparent metalsfor ultrabroadband electromagnetic waves," Advanced Materials, 2012, 24(15): 1980-1986.

[21] N. K. Grady, J. E. Heyes, D. R. Chowdhury, Y. Zeng, M. T. Reiten, A. K. Azad, et al., "Terahertz metamaterials for linear polarization conversion and anomalous refraction," Science, 2013, 340(6138): 1304-1307. 
[22] I. Kogelbauer, E. Heine, C. D'Amboise, C. Müllebner, W. Sokol, and W. Loiskandl, "Adaptation of soil physical measurement techniques for the delineation of mud and lakebed sediments at neusiedler see," Sensors, 2013, 13(12): 17067-17083.

[23]C. Fevillet-Palma, Y. Todorov, R. Steed, A. Vasanelli, G. Biasiol, L. Sorba, et al., "Extremely sub-wavelength $\mathrm{THz}$ metal-dielectric wire microcavities," Optics Express, 2012, 20(27): 29121-29130.

[24] Y. Todorov, L. Tosetto, J. Teissier, A. M. Andrews, P. Klang, R. Colombelli, et al., "Optical properties of metal-dielectric-metal microcavities in the $\mathrm{THz}$ frequency range," Optics Express, 2010, 18(13): 13886-13907.
[25] C. Fevillet-Palma, Y. Todorow, A. Vasanelli, and C. Sirtori, "Strong near field enhancement in $\mathrm{THz}$ nano-antenna arrays," Sientific Reports, 2013, 3(1): 299-308.

[26] X. D. Wang, Y. H. Ye, C. Zhang, Y. Qin, and T. J. Cui, "Tunable figure of merit for a negative-index metamaterial with a sandwich configuration," Optics Letters, 2009, 34(22): 3568-3570.

[27] K. Chen, Q. Y. Wen, and H. B. Znang, "Study on the broadband terahertz metamaterial absorber," Electronic Components and Materials, 2011, 30(7): 56-59.

[28] N. I. Landy, S. Sajuyigbe, J. J. Mock, D. R. Smith, and W. J. Padilla, "Perfect metamaterial absorber," Physical Review Letters, 2008, 100(20): 1586-1594. 